\title{
Denoising MAX6675 reading using Kalman filter and factorial design
}

Reski Septiana, Ibnu Roihan, Raldi A. Koestoer

Department of Mechanical Engineering, Universitas Indonesia, Indonesia

\begin{tabular}{l}
\hline \hline Article Info \\
\hline Article history: \\
Received Oct 25, 2020 \\
Revised Mar 27, 2021 \\
Accepted Apr 11, 2021 \\
\hline
\end{tabular}

\section{Keywords:}

Kalman filter

MAX6675

Tuning input variables

Tuning KF

Two-level factorial design

\begin{abstract}
This paper aims to tune the Kalman filter (KF) input variables, namely measurement error and process noise, based on two-level factorial design. Kalman filter then was applied in inexpensive temperature-acquisition utilizing MAX6675 and K-type thermocouple with Arduino as its microprocessor. Two levels for each input variable, respectively, 0.1 and 0.9 , were selected and applied to four K-type thermocouples mounted on MAX6675. Each sensor with a different combination of input variables was used to measure the temperature of ambient-water, boiling water, and sudden temperature drops in the system. The measurement results which consisted of the original and KF readings were evaluated to determine the optimum combination of input variables. It was found that the optimum combination of input variables was highly dependent on the system's dynamics. For systems with relatively constant dynamics, a large value of measurement error and small value of process noise results in higher precision readings. Nevertheless, for fast dynamic systems, the previous input variables' combination is less optimal because it produced a time-gap, which made the $\mathrm{KF}$ reading differ from the original measurement. The selection of the optimum input combination using two-level factorial design eased the KF tuning process, resulting in a more precise yet low-cost sensor.
\end{abstract}

This is an open access article under the CC BY-SA license.

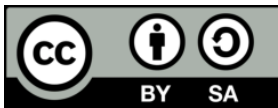

Corresponding Author:

Raldi Artono Koestoer

Department of Mechanical Engineering

Universitas Indonesia

Kampus UI Depok, Indonesia

Email: koestoer@eng.ui.ac.id

\section{INTRODUCTION}

In response to the rapid development of technology, the information and knowledge nowadays are freely accessible around the world as indicated by massive open access and open source platforms. Arduino, one of the open-source platforms, eases many people, including makers and scientists to acquire data and control things. Microcontroller like Arduino breaks the obstacle of expensive commercial instruments such as data acquisition (DAQ) [1], [2]. Economical Arduino-DAQ can easily be made to monitor the system with the help of low-cost sensors and modules. However, as the response of vast deployment of low-cost sensor and module [3], the resulted measurements are corrupted with noise [4] which need to be filtered first to know the real value of the parameter being measured.

From so many filtering techniques out there, Kalman filter (KF) has been widely applied in many fields, because of its simple algorithm, good filter quality in reducing variance, does not take up much memory, and can be applied in real-time [5]-[8]. However, to get the optimum filter results from KF, the three initial statistical assumptions or herein called the three input variables, namely estimation error, process 
noise and measurement noise must be tuned correctly because an incorrect combination can result in imprecise readings or produce readings that misrepresent the state of system [5], [9]-[11]. In practical applications, the noise covariances, especially process and measurement noise are generally not known, therefore, choosing or tuning the value of measurement and process noise covariances is considered a perplexing task [12]. Many papers have discussed about the tuning process of Kalman filter variables, Korniyenko et al. proposed neural-network based approach for tuning the KF [13], while Loebis et al. proposed adaptive tuning using fuzzy logic approach [14], also Ting et al. and Aleti et al. proposed an approach based on genetic algorithm [15], [16]. All of the presented methods are considered highly sophisticated, which required the readers to fully understand how to code the approach to be applied directly to the sensors. To fully taking advantage of the Kalman Filter to give optimal readings without pre-requisite skills or background, such as advance coding skill or computer science background, two-level factorial design can be employed for KF tuning problem. Although the proposed method is considered conventional and not adaptive, the method is robust, simple, and can sufficiently portray the decision steps that should be done regarding the tuning process.

The factorial design has been successfully implemented in various studies to evaluate the effect of various parameters which resulting in optimum objective [17]-[20]. The use of factorial design in KF tuning problem not only can assist people with limited coding skills, but also can ease the limitation of neural network $(\mathrm{NN})$ based approach in reducing the number of data sets. The $\mathrm{NN}$ can be trained properly with essential yet minimized data sets to avoid the lack of generalization capability, thus reducing the computational time. This paper aims to assist the tuning process of KF based on two-level factorial design to be applied in low-cost temperature-acquisition using Arduino and MAX6675 module. Recently, MAX6675 has become more common to be used as cold-junction compensation for K-type thermocouple [2], [21]-[23]. Yet the performance evaluation of the sensor has not been reported. In line with our objective, the performance evaluation of the sensor was also discussed here as a basis to analyze which optimum combination best-represent the system and sensor's characteristics.

\section{LITERATURE REVIEW}

\subsection{Kalman filter}

Kalman filter (KF) is an algorithm which estimates the true value of hidden variable based on previous measurement which full of noise and inaccuracy [5], [24]. KF combines the predicted states and noisy measurements to produce optimal and unbiased estimates of the system parameter. This algorithm is widely used for prediction or estimation, but is called a filter because of its optimal ability to minimize variance [25]. According to Mohinder and Angus, Kalman Filter is possibly the greatest discovery in the 20th century [26], because of its application that has been widely used in the inertial navigation system [27]-[33], freeway traffic modelling [34]-[37], object tracking [38]-[42], image processing [43]-[48], even modelling the marine ecosystem [49]. The basic principle of the Kalman algorithm is determined by (1) and (2). Kalman models the output measurement of the sensor $(y)$ at a certain time $(t)$ as a linear function of the parameter of interest $(x)$ combined with the conversion matrix $(H)$ as a measurement converter accompanied by measurement noise $(v)$.

$$
y_{t}=H_{t} x_{t}+v_{t}
$$

The dynamic of the parameter of interest $(x)$ at time $(t)$ is modelled as a linear combination of the previous state $(x$ at $t-1)$ combined with the transition matrix $(F)$, the input control $(u)$ and its transition matrix $(G)$, as well as process noise $(w)$.

$$
x_{t}=F_{t-1} x_{t-1}+G_{t-1} u_{t-1}+w_{t-1}
$$

To distinguish between measurement noise $(v)$ and the actual value of the parameter of interest $(x)$ from a sensor reading $(y)$, the Kalman algorithm uses several mathematical equations listed in Table 1. The block diagram of Kalman Filter's algorithm is shown in Figure 1. Based on the schematic in Figure 1, the system must be modelled priorly with three input variables, namely estimation error $(p)$, process noise $(w)$ and measurement error $(v)$ before KF can filter noise from output measurement using (3)-(6). Estimation error $(p)$ is an initial estimation of the measured parameter. The value of this variable can be filled with any number because KF will adjust the value to the actual condition based on the sensor measurement results.

Process noise $(w)$ relates to system dynamics or often is illustrated by how fast the state of the system changes. The value of this variable is in the range of 0 to 1 . If this variable is equal to 0 then the system is modelled to be in constant or stable condition. Meanwhile, if this variable has a value of 1 then the 
system is modelled to have a very fast dynamics or tends to change rapidly. Measurement error $(v)$ is a variable related to measurement variance. Because the measurement results are closely related to the sensor used, this variable is usually filled with the uncertainty of the sensors used.

Table 1. Important equations in Kalman filter's algorithm [50]

\begin{tabular}{ccc}
\hline Name & Equation \\
\hline Kalman Gain as control variable (weight equation) & $K G_{t}=\frac{p_{t-1}}{p_{t-1}+v_{t}}$ \\
Current State Estimation as filtering equation & $x_{t}=x_{t-1}+K G_{t}\left(y_{t}-x_{t-1}\right)$ \\
Uncertainty of Current State Estimation & $p_{t}=\left(1-K G_{t}\right) p_{t-1}+w_{t}\left|x_{t-1}-x_{t}\right|$ \\
Update Estimate as transition equation & $x_{t-1}=x_{t}$
\end{tabular}

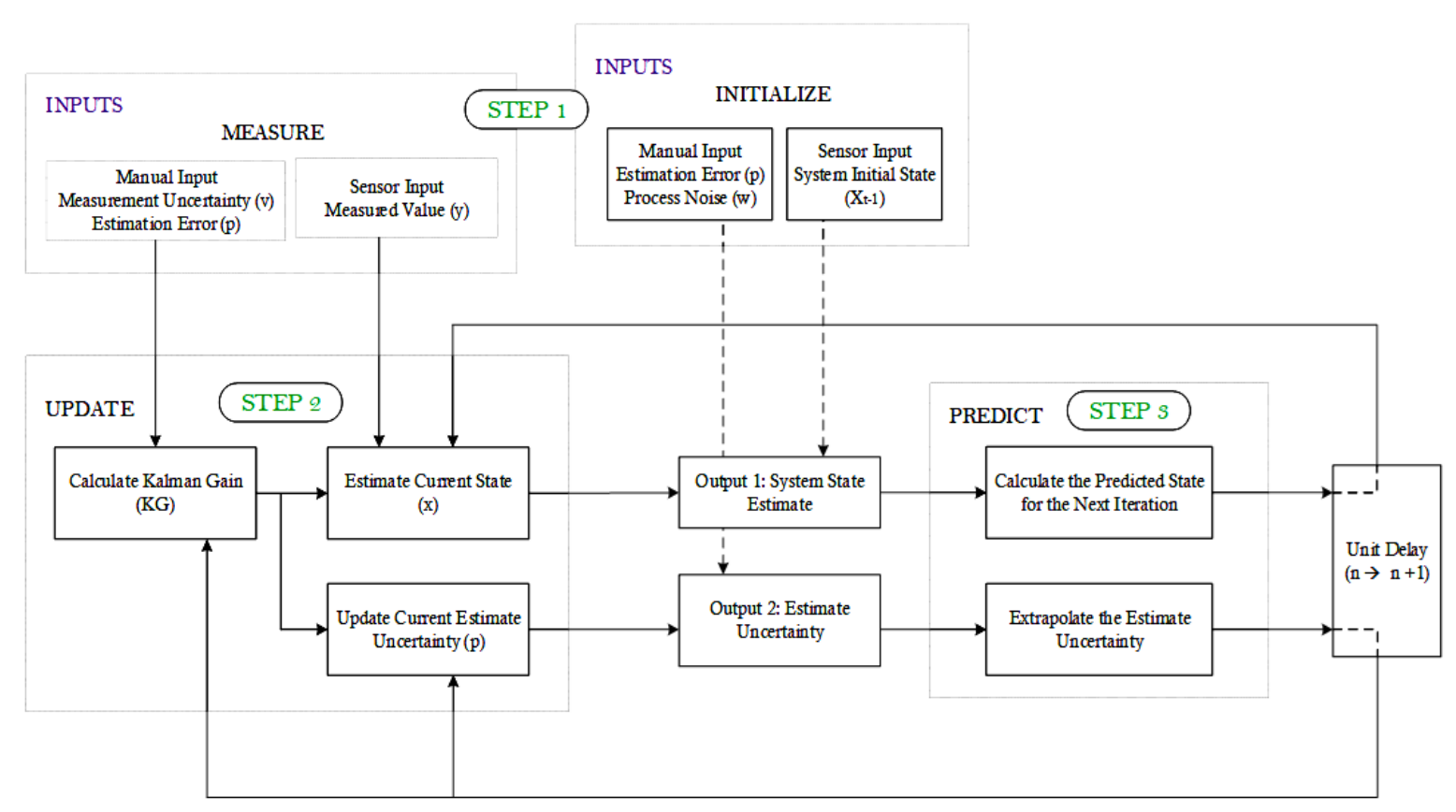

Figure 1. Algorithm of Kalman filter [50]

\section{METHOD}

The Kalman filter was applied to four K-type thermocouples mounted on MAX6675 to measure the ambient water temperature for 24 hours, the boiling water temperature, and sudden change of temperature in the system. Ambient water was used as a comparison to the natural low-dynamics system, while water in the boiling process is categorized as the unnatural low-dynamics system. The sudden change of the system's temperature is a simulation of a system-failure which categorized as fast dynamics system. The fast dynamics system is a modification of the low-dynamics system where the four sensors that were measuring the boiling water temperature suddenly released into the ambient air. The experimental set-up of this experiment can be seen in Figure 2. The four sensors were first used to measure the temperature of ambient water for 24 hours, then the heater was turned on to boil the water with 100 Watt power and 200 Volt input voltage which already been stabilized by the regulator. After the water was boiling for a while, the four sensors were exposed to ambient air. The four K-type thermocouples and MAX6675 sensors were connected to Arduino Mega as the microprocessor to input both the measurement commands and the algorithm of Kalman filter as in (3)-(6). The four sensors measured the system temperature every $100 \mathrm{~ms}$ to be stored as text in the SD card. Compatibility of Arduino and SD Card creates an inexpensive data-logger [1], [51] which reduce electricity consumption.

Each K-type thermocouple mounted on MAX6675 sensor has different values of KF's input variables, which can be seen in the second to fourth columns of Table 2. All sensors were placed relatively near to each other in the same system, so all sensors were set to have the same value of estimation error, $10{ }^{\circ} \mathrm{C}$. The estimation error value was set far from the ambient water temperature to see resilience of the filter or how long the filter results converge withf the actual temperature as measured by the sensor. In order to 
select the optimal combination of input variables to produce a smaller deviation, two of the three Kalman input variables, namely the process noise and measurement errors will be analyzed using two-level factorial designs. The same value of high and low level, respectively 0.9 and 0.1 , was selected for each variable, as conservative estimates which fall within the range of process noise value, 0 to 1 , but not the exact 0 or 1 because mostly we don't really know the actual state of the system during observation. Although the uncertainty of K-type thermocouple mounted on MAX6675 sensor based on previous study was between $0.22{ }^{\circ} \mathrm{C}$ to $0.26{ }^{\circ} \mathrm{C}[21]$, the sensor uncertainty in this study was set to 0.1 and 0.9 to see the effect of the unstandardized values of measurement error to the filter results.

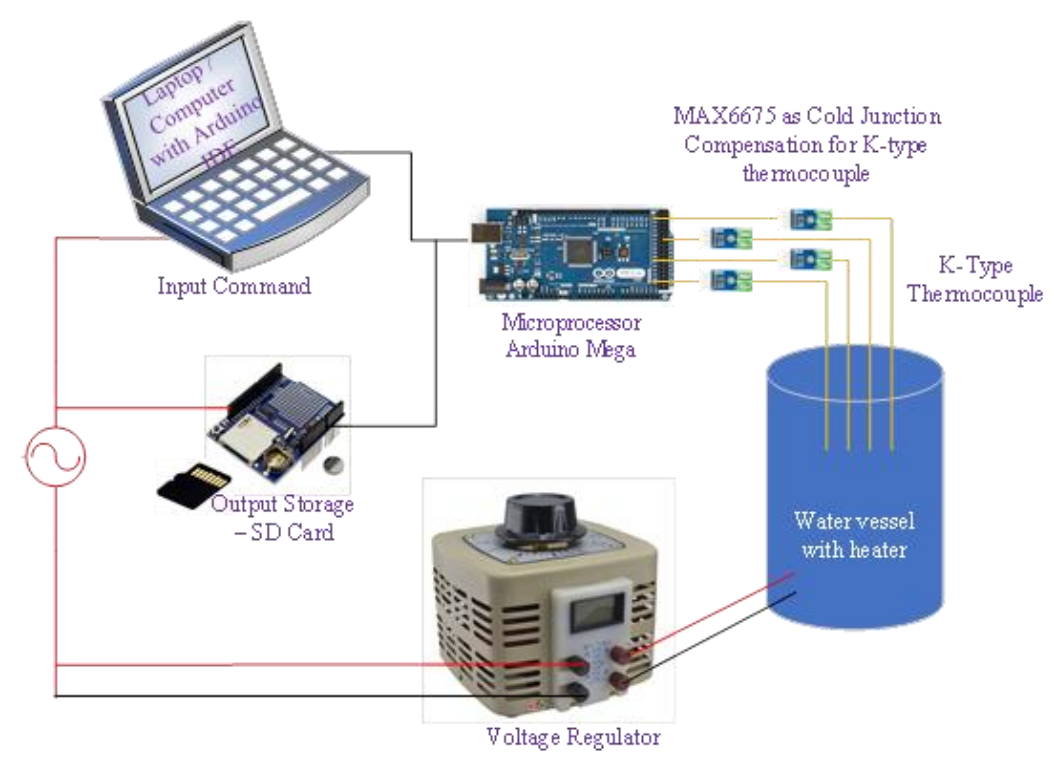

Figure 2. Experimental set-up

Table 2. Input variables and its resulting deviation of each sensor

\begin{tabular}{cccccc}
\hline Sensor no- & Estimation error & Measurement error & Process noise & Before employing KF & After employing KF \\
\hline 1 & 10 & 0.9 & 0.1 & 0.25 & 0.02 \\
2 & 10 & 0.1 & 0.9 & 0.22 & 0.15 \\
3 & 10 & 0.1 & 0.1 & 0.22 & 0.07 \\
4 & 10 & 0.9 & 0.9 & 0.25 & 0.09 \\
\hline
\end{tabular}

\section{RESULTS AND DISCUSSION}

The results of each sensor's original reading and KF's reading in ambient water temperature for 24 hours are shown in Figure 3. The original readings of each sensor (TC-i) are shown in black line, while the Kalman filter's readings (TCF-i) are shown in red. All the four sensors of K-type thermocouples mounted on MAX6675 were placed relatively close to each other and came from the same manufacturer, but the accuracy of each sensor is slightly different between one another. The four of them showed the same trend in reading the dynamics of water temperature for 24 hours, but with big oscillation. The difference between measured value at time and could reach up to $1.5^{\circ} \mathrm{C}$, even though the datasheet of MAX6675 stated that the noise already been filtered in the internal circuit of the sensor [52].

Based on Figure 3, Kalman algorithm worked well in filtering the original readings of sensors, but the results differ between sensors because the performance is very dependent on the input variable as in Table 2. The KF's input variables for the first sensor produced the smallest deviation among the other. While the input variables for the second sensor produced filter results that hardly differ from its original reading. The KF's input variables for the third and fourth sensors produced filter results that appear to be the same to each other, with deviations smaller than the original deviations of the sensors but greater than the deviation generated by the KF's input variables on the first sensor. The calculated deviations before and after filtering are shown in the last two columns of Table 2.

As shown in Figure 3, the KF results on the first and fourth sensors did not immediately converge to the sensors' output measurement at the beginning. The algorithm on those two sensors took approximately one second to converge to the original output measurement. This is most likely due to a large value of 
measurement error variable so the Kalman gain value was smaller on both sensors which makes the KF algorithm trust the output measurement less than the calculated estimate value. The relationship between the deviation produced and the input variable of each sensor was analysed using two-level factorial design, as shown in Figure 4. Sensor 1 to sensor 4 is denoted by S1 to S4, with the value of input variables and its resulting deviation based on Table 2 . If the level of measurement error was increased, the average deviation decreased around $0.055^{\circ} \mathrm{C}$. This indirectly indicates that the $\mathrm{KF}$ algorithm knows that the greater the sensor's measurement error, the measurement input given by the sensor is very noisy so the resulting filter became more precise. The deviation was increased by approximately $0.075^{\circ} \mathrm{C}$ if the level of the process noise was increased. This is reasonable, given the system was in ambient state which doesn't tend to change drastically, so the smaller the value of the process noise, the better it is in modelling the real system dynamics which affects to the smaller deviation produced by KF. The interaction effect of the two input variables was less significant in changing the KF deviation in a natural low-dynamic system, only $0.005{ }^{\circ} \mathrm{C}$. For natural and low-dynamic systems, simply reducing the value of the process noise will result in a more precise reading.
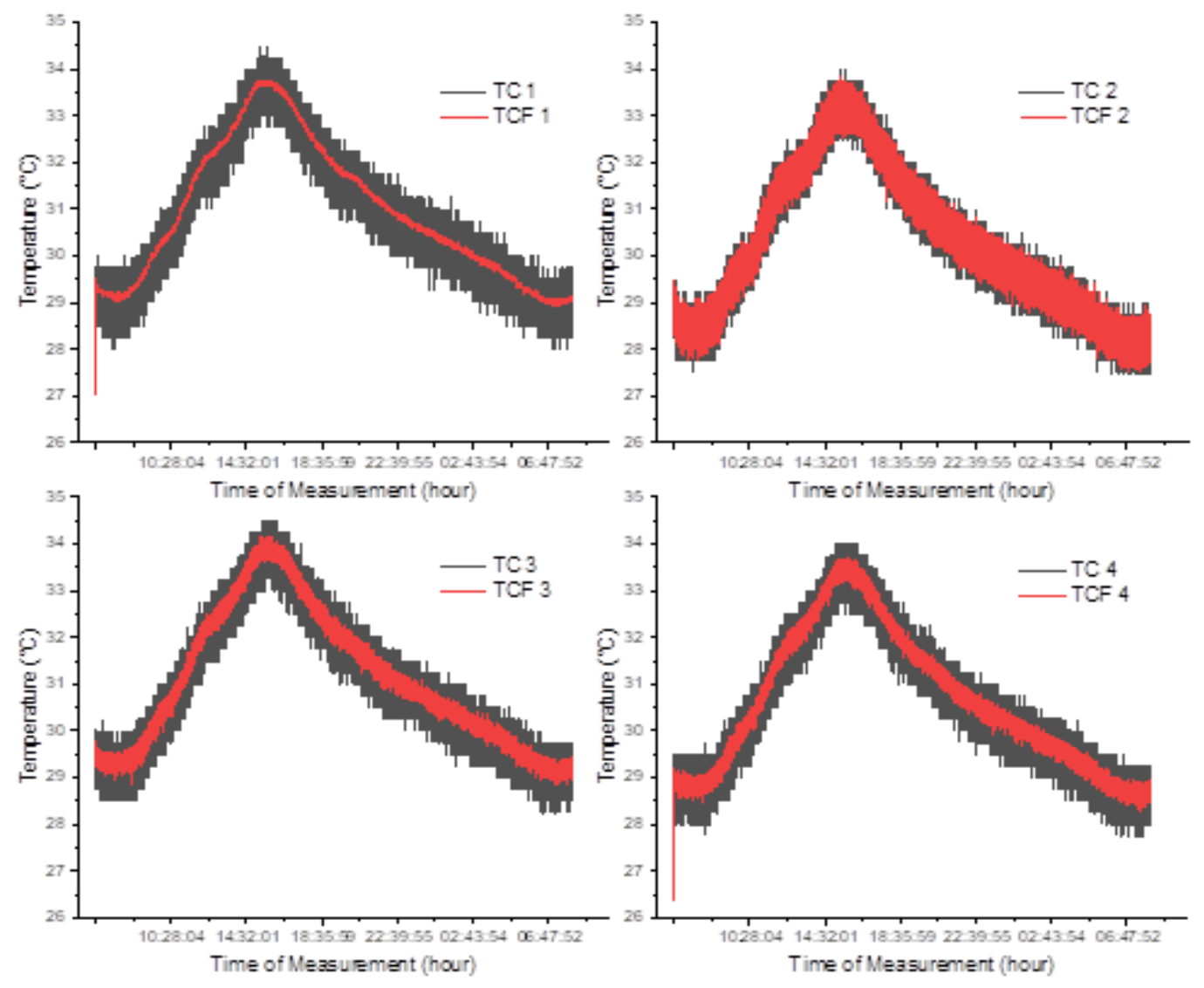

Figure 3. Original and filtered readings of each sensor in water ambient temperature for 24 hours

The performance of Kalman Filter in reading the temperature changes in boiling process is shown in Figure 5, which consists of a close-up view of the boiling process. The results of KF readings in this process were almost same with the readings in ambient water. The input variables of the first sensor produced the smallest deviation between the other input variables and the input variables of the second sensor produced a filter reading that is almost the same to the original sensor reading. The effect of the input variables to the deviation produced by KF in reading the temperature changes in boiling process using two-level factorial design is shown in Figure 6. Compared to Figure 4, only the effect of measurement error variable that has not changed. This indicates the independency of sensor's measurement error to the state of system. The effect of process noise which was smaller than the value calculated on natural low-dynamics system, indirectly indicates that Kalman Filter algorithm knows that the system being measured has different dynamics from the previous system. For systems with additional input energy from the outside, although changing the value of the process noise could change the deviation of the KF reading, it was less significant than when the 
system is in a natural state. A significant change in deviation will occur when the measurement error value is greater. The interaction between the two variables had a greater influence in changing the sensor deviation than the results obtained in ambient conditions.

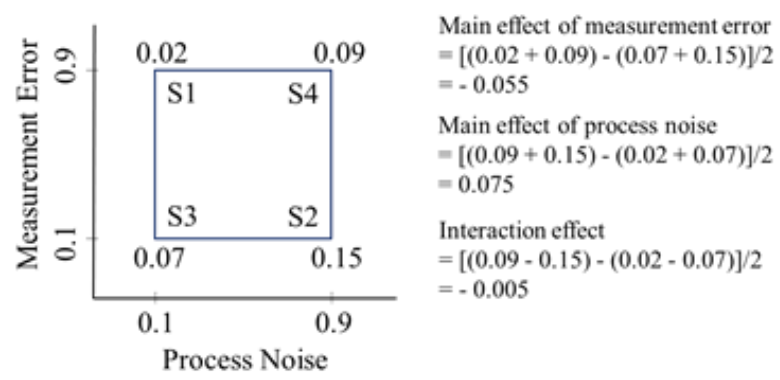

Figure 4. Respond deviation of each sensor in reading ambient water temperature using two-level factorial design
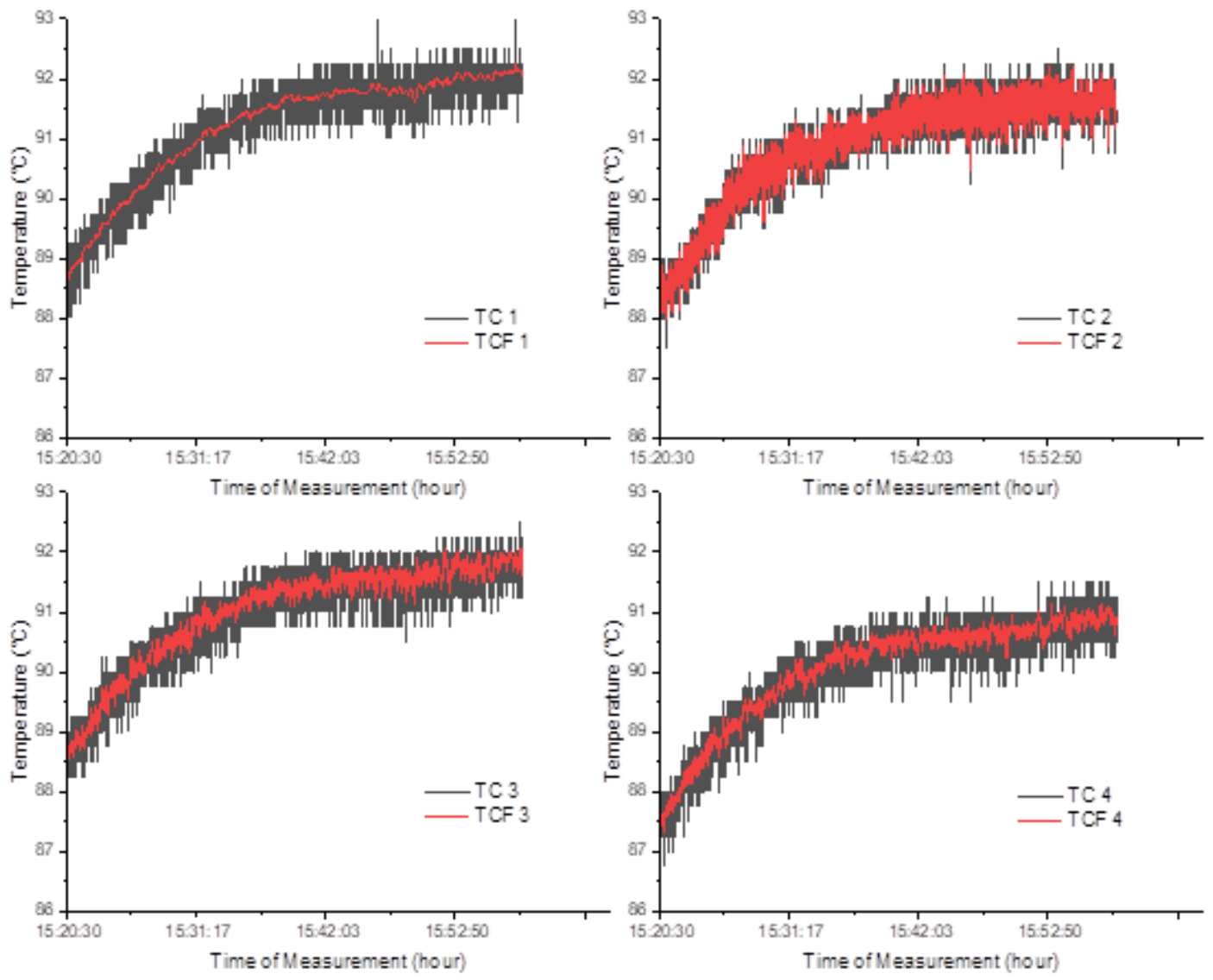

Figure 5. Close-up view measurement readings of each sensor in boiling water temperature

For low dynamics systems, large value of measurement error and small value of process noise were possibly the optimum combination of KF input variables to produce more precise readings. Measurement error values that were not in accordance with sensor specifications also did not affect KF performance. The performance of the four combinations of KF input variables in reading sudden temperature changes is shown in Figure 7. When there is a swift temperature drop, the first sensor with large value of measurement error and small value of process noise produced a filter result that somewhat different from the original sensor readings. A time-gap or delay around one second existed before the KF reading on the first sensor adjusted its dynamics to the sensor's original measurement. In a fast-dynamic system, the factorial design analysis 
regarding input variables influenced the deviation produced was somehow inappropriate because of the delay. The response time of each KF input variable was analyzed instead. Graphically, the combination of input variables for the second sensor produced the same dynamic between KF results and the original output measurement from sensor, therefore deviation resulted were not much different from the original sensor's reading. The KF readings on the third and fourth sensors illustrated the real-time dynamics of the sensors without delay with smaller deviations. Hence, it indicated the efficacy of KF algorithm in filtering the original measurement with the proposed input variables. The deviation produced by KF on the third and fourth sensors was smaller than the original deviation of the sensor but not as precise as the KF results on the first sensor. Referring back to Figure 3, the combination of input variables on the fourth sensor required a warm-up time or adjustment time when used for the first time. This can be neglected with changing the value of estimation error as close to real condition. However, it can be a consideration in choosing the right combination of input variables.

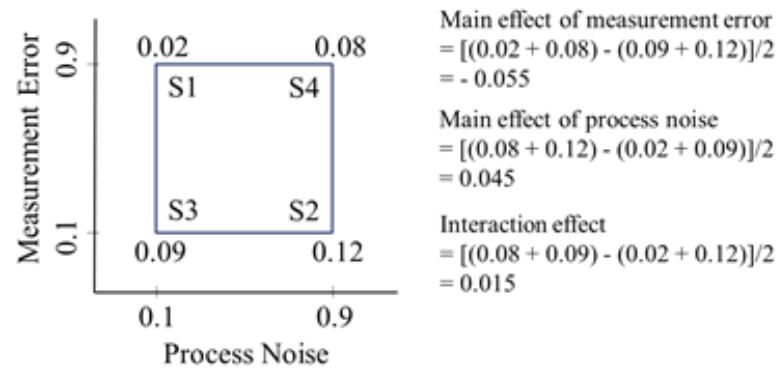

Figure 6. Respond deviation of each sensor in reading boiling water temperature using two-level factorial design
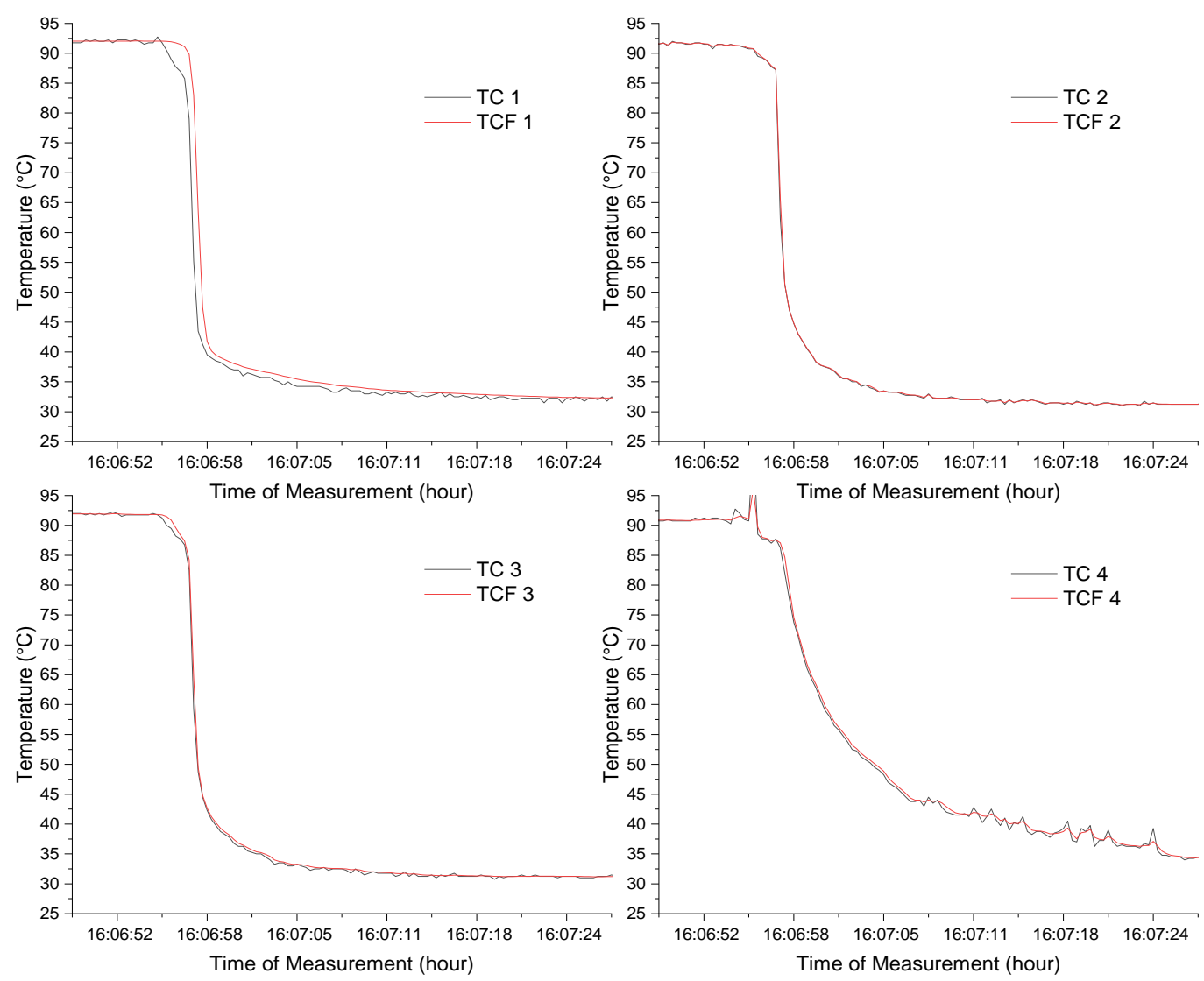

Figure 7. Performance of each sensor in reading sudden temperature's change 


\section{CONCLUSION}

The performance of the four pairs of MAX6675 and K-type thermocouple using Arduino as temperature-acquisition have been evaluated. All of the sensors showed great tendency to be used as inexpensive DAQ because they produced the same trend in reading temperature from ambient to almost $10^{\circ} \mathrm{C}$. However, they produced different readings between each other, which compromise the accuracy of the sensors. Further calibration process is recommended to make the temperature acquisition based on MAX6675 and K-type thermocouple more valid. The output measurements resulting from K-type thermocouple and MAX6675 also have huge oscillations, around $0.22-0.25^{\circ} \mathrm{C}$. Therefore, the filtering process, such as Kalman Filter, was needed to make the sensors' readings more precise.

The application of Kalman filter on K-type thermocouples and MAX6675 sensor produced fairly precise real-time readings when used with the right combination of input variables. The two-level factorial design could simplify the tuning process of the input variables by looking at the main effect and the interaction effect between variables. In the constant-dynamic systems, a combination of large value of measurement error and small value of process noise resulted in a more precise reading with small deviation of $0.02{ }^{\circ} \mathrm{C}$. However, the previous combination produced a time-gap or delay when applied to a fast-dynamic system. The delay slows the filter results in responding to the system's real dynamics. For systems with fast dynamics, it would be better to use a combination of measurement error and process noise values at the same level because the filter readings adequately represented the actual system dynamics without delay and produced smaller deviations than the sensor's original measurement, around $0.07-0.09^{\circ} \mathrm{C}$.

\section{ACKNOWLEDGEMENTS}

This work was supported by the Ministry of Research, Technology and Higher Education of the Republic of Indonesia (PMDSU Grant NKB-446/UN2.RST/HKP.05.00/2020). We are grateful to the anonymous referee for constructive suggestions.

\section{REFERENCES}

[1] P. A. Beddows and E. K. Mallon, "Cave Pearl Data Logger: A Flexible Arduino-Based Logging Platform for Long-Term Monitoring in Harsh Environments," (in English), Sensors (Basel), vol. 18, no. 2, 2018, Art. no. 530, doi: $10.3390 / \mathrm{s} 18020530$.

[2] S. P. Nalavade, A. D. Patange, C. L. Prabhune, S. S. Mulik, and M. S. Shewale, "Development of 12 Channel Temperature Acquisition System for Heat Exchanger Using MAX6675 and Arduino Interface," Singapore, 2019: Springer Singapore, in Innovative Design, Analysis and Development Practices in Aerospace and Automotive Engineering (I-DAD 2018), pp. 119-125, 2018, doi: 10.1007/978-981-13-2697-4_13.

[3] Z. Wu, M. Fu, Y. Xu, and R. Lu, "A distributed Kalman filtering algorithm with fast finite-time convergence for sensor networks," Automatica, vol. 95, pp. 63-72, 2018, doi: 10.1016/j.automatica.2018.05.012.

[4] R. Ferrero, F. Gandino, and M. Hemmatpour, "Estimation of displacement for Internet of Things applications with Kalman filter," Electronics, vol. 8, no. 9, 2019, Art. no. 985, doi: 10.3390/electronics8090985.

[5] K. Youngjoo and B. Hyochoong, "Introduction to Kalman Filter and Its Applications," Introduction and Implementations of the Kalman Filter, 2019, doi: 10.5772/intechopen.80600.

[6] R. G. Valenti, I. Dryanovski, and J. Xiao, "A linear Kalman filter for MARG orientation estimation using the algebraic quaternion algorithm," IEEE Transactions on Instrumentation and Measurement, vol. 65, no. 2, pp. 467-481, 2016, doi: 10.1109/TIM.2015.2498998.

[7] S. Wang, W. Wang, B. Chen, and K. T. Chi, "Convergence analysis of nonlinear Kalman filters with novel innovation-based method," Neurocomputing, vol. 289, pp. 188-194, 2018, doi: 10.1016/j.neucom.2018.02.001.

[8] D. Cherkassky and S. Gannot, "New insights into the Kalman filter beamformer: Applications to speech and robustness," IEEE Signal Processing Letters, vol. 23, no. 3, pp. 376-380, 2016, doi: 10.1109/LSP.2016.2519859.

[9] S. Mohan M., N. Naik, R. M. O. Gemson, and M. R. Ananthasayanam, "Introduction to the Kalman Filter and Tuning its Statistics for Near Optimal Estimates and Cramer Rao Bound," Indian Institute of Technology Kanpur, India, 2015. [Online]. Available: https://arxiv.org/pdf/1503.04313.pdf

[10] R. A. Mudambi, "Tuning of the Kalman Filter Using Constant Gains," Introduction and Implementations of the Kalman Filter, 2019, doi: 10.5772/intechopen.81795.

[11] S. Khashirunnisa, B. K. Chand, and B. L. Kumari, "Performance analysis of Kalman filter, fuzzy Kalman filter and wind driven optimized Kalman filter for tracking applications," in 2016 2nd International Conference on Communication Control and Intelligent Systems (CCIS), 2016, pp. 170-174, doi: 10.1109/CCIntelS.2016.7878223.

[12] B. M. Åkesson, J. B. Jørgensen, N. K. Poulsen, and S. B. Jørgensen, "A tool for kalman filter tuning," in Computer Aided Chemical Engineering, vol. 24, pp. 859-864, 2007, doi: 10.1016/S1570-7946(07)80166-0.

[13] O. V. Korniyenko, M. S. Sharawi, and D. N. Aloi, "Neural Network Based Approach for Tuning Kalman Filter," in 2005 IEEE International Conference on Electro Information Technology, 2005, pp. 1-5, doi: 10.1109/EIT.2005.1626991. 
[14] D. Loebis, R. Sutton, J. Chudley, and W. Naeem, "Adaptive tuning of a Kalman filter via fuzzy logic for an intelligent AUV navigation system," Control engineering practice, vol. 12, no. 12, pp. 1531-1539, 2004, doi: 10.1016/j.conengprac.2003.11.008.

[15] T. Ting, K. L. Man, E. G. Lim, and M. Leach, "Tuning of Kalman filter parameters via genetic algorithm for stateof-charge estimation in battery management system," The Scientific World Journal, vol. 2014, 2014, doi: $10.1155 / 2014 / 176052$.

[16] A. Aleti and L. Grunske, "Test data generation with a Kalman filter-based adaptive genetic algorithm," Journal of Systems and Software, vol. 103, pp. 343-352, 2015, doi: 10.1016/j.jss.2014.11.035.

[17] N. D. Burrows, S. Harvey, F. A. Idesis, and C. J. Murphy, "Understanding the seed-mediated growth of gold nanorods through a fractional factorial design of experiments," Langmuir, vol. 33, no. 8, pp. 1891-1907, 2017 , doi: 10.1021/acs.langmuir.6b03606.

[18] T. A. Saleh, M. Tuzen, and A. Sar1, "Polyamide magnetic palygorskite for the simultaneous removal of $\mathrm{Hg}$ (II) and methyl mercury; with factorial design analysis," Journal of environmental management, vol. 211, pp. 323-333, 2018, doi: 10.1016/j.jenvman.2018.01.050.

[19] S. O. Adio, M. H. Omar, M. Asif, and T. A. Saleh, "Arsenic and selenium removal from water using biosynthesized nanoscale zero-valent iron: a factorial design analysis," Process Safety and Environmental Protection, vol. 107, pp. 518-527, 2017, doi: 10.1016/j.psep.2017.03.004.

[20] M. H. Esfe, H. Rostamian, A. Shabani-Samghabadi, and A. A. A. Arani, "Application of three-level general factorial design approach for thermal conductivity of $\mathrm{MgO} /$ water nanofluids," Applied Thermal Engineering, vol. 127, pp. 1194-1199, 2017, doi: 10.1016/j.applthermaleng.2017.07.211.

[21] R. Septiana, I. Roihan, and R. A. Koestoer, "Testing a Calibration Method for Temperature Sensors in Different Working Fluids," Journal of Advanced Research in Fluid Mechanics and Thermal Sciences, vol. 68, no. 2, pp. 84-93, 2020, doi: 10.37934/arfmts.68.2.8493.

[22] F. A. Obando Vega, A. P. Montoya Ríos, J. A. Osorio Saraz, L. G. Vargas Quiroz, and F. Alves Damasceno, "Assessment of black globe thermometers employing various sensors and alternative materials," Agricultural and Forest Meteorology, vol. 284, p. 107891, 2020, doi: 10.1016/j.agrformet.2019.107891.

[23] T. Shimada, T. Miura, W. Xie, T. Yanase, and T. Nagahama, "A thermocouple-based remote temperature controller of an electrically-floated sample for plasma CVD of nanocarbons with bias voltage," Measurement, vol. 102, pp. 244-248, 2017, doi: 10.1016/j.measurement.2017.02.012.

[24] Q. Li, R. Li, K. Ji, and W. Dai, "Kalman filter and its application," in 2015 8th International Conference on Intelligent Networks and Intelligent Systems (ICINIS), 2015, pp. 74-77, doi: 10.1109/ICINIS.2015.35.

[25] Y. Huang, Y. Zhang, Y. Zhao, and J. A. Chambers, "A Novel Robust Gaussian-Student's t Mixture Distribution Based Kalman Filter," IEEE Transactions on Signal Processing, vol. 67, no. 13, pp. 3606-3620, 2019, doi: 10.1109/TSP.2019.2916755.

[26] M. Grewal and A. Andrews, "Kalman filtering: theory and practice using MATLAB," New York: John Wiley and Sons, vol. 14, 2001, doi: 10.1002/9780470377819.

[27] Y. Ning, J. Wang, H. Han, X. Tan, and T. Liu, "An optimal radial basis function neural network enhanced adaptive robust Kalman filter for GNSS/INS integrated systems in complex urban areas," Sensors, vol. 18, no. 9, 2018, Art. no. 3091, doi: 10.3390/s18093091.

[28] B. Cui, X. Wei, X. Chen, J. Li, and L. Li, "On sigma-point update of cubature Kalman filter for GNSS/INS under GNSS-challenged environment," IEEE Transactions on Vehicular Technology, vol. 68, no. 9, pp. 8671-8682, 2019, doi: 10.1109/TVT.2019.2931923.

[29] Y. Liu, X. Fan, C. Lv, J. Wu, L. Li, and D. Ding, "An innovative information fusion method with adaptive Kalman filter for integrated INS/GPS navigation of autonomous vehicles," Mechanical Systems and Signal Processing, vol. 100, pp. 605-616, 2018, doi: 10.1016/j.ymssp.2017.07.051.

[30] K. Feng et al., "An improved strong tracking cubature Kalman filter for GPS/INS integrated navigation systems," Sensors, vol. 18, no. 6, 2018, Art. no. 1919, doi: 10.3390/s18061919.

[31] B. Cui, X. Chen, and X. Tang, "Improved cubature Kalman filter for GNSS/INS based on transformation of posterior sigma-points error," IEEE Transactions on Signal Processing, vol. 65, no. 11, pp. 2975-2987, 2017, doi: 10.1109/TSP.2017.2679685.

[32] C. Shen, Y. Zhang, J. Tang, H. Cao, and J. Liu, "Dual-optimization for a MEMS-INS/GPS system during GPS outages based on the cubature Kalman filter and neural networks," Mechanical Systems and Signal Processing, vol. 133, 2019, Art. no. 106222, doi: 10.1016/j.ymssp.2019.07.003.

[33] L. Zhao, H. Qiu, and Y. Feng, "Analysis of a robust Kalman filter in loosely coupled GPS/INS navigation system," Measurement, vol. 80, pp. 138-147, 2016, doi: 10.1016/j.measurement.2015.11.008.

[34] A. Ouessai and M. Keche, "Real-Time Freeway Traffic State Estimation Based on the Second-Order Divided Difference Kalman Filter," Transport and Telecommunication Journal, vol. 20, no. 2, pp. 114-122, 2019, doi: 10.2478/ttj-2019-0010.

[35] Y. Liu, S. He, B. Ran, and Y. Cheng, "A progressive extended Kalman filter method for freeway traffic state estimation integrating multisource data," Wireless Communications and Mobile Computing, vol. 2018, pp. 1-10, 2018, doi: 10.1155/2018/6745726.

[36] C. Wang, B. Ran, H. Yang, J. Zhang, and X. Qu, "A novel approach to estimate freeway traffic state: parallel computing and improved Kalman filter," IEEE Intelligent Transportation Systems Magazine, vol. 10, no. 2, pp. 180-193, 2018, doi: 10.1109/MITS.2018.2806627. 
[37] H. Yang, P. J. Jin, B. Ran, D. Yang, Z. Duan, and L. He, "Freeway traffic state estimation: A Lagrangian-space Kalman filter approach," Journal of Intelligent Transportation Systems, vol. 23, no. 6, pp. 525-540, 2019, doi: 10.1080/15472450.2018.1476147.

[38] C. K. Chui and G. Chen, "Kalman filtering," in Springer Series in Information Sciences, 5 ed.: Springer International Publishing, 2017, pp. XVIII, 247.

[39] R. Ferrero, F. Gandino, M. Hemmatpour, B. Montrucchio, and M. Rebaudengo, "Exploiting accelerometers to estimate displacement," in 2016 5th Mediterranean Conference on Embedded Computing (MECO), 2016, pp. 206-210, doi: 10.1109/MECO.2016.7525741.

[40] Z. Cai, Z. Gu, Z. L. Yu, H. Liu, and K. Zhang, "A real-time visual object tracking system based on Kalman filter and MB-LBP feature matching," Multimedia Tools and Applications, vol. 75, no. 4, pp. 2393-2409, 2016, doi: 10.1007/s11042-014-2411-6.

[41] Z. Zhou, D. Wu, and Z. Zhu, "Object tracking based on Kalman particle filter with LSSVR," Optik, vol. 127, no. 2, pp. 613-619, 2016, doi: 10.1016/j.ijleo.2015.10.038.

[42] H. Ait Abdelali, F. Essannouni, L. Essannouni, and D. Aboutajdine, "An adaptive object tracking using Kalman filter and probability product kernel," Modelling and Simulation in Engineering, vol. 2016, 2016, doi: $10.1155 / 2016 / 2592368$.

[43] L. Wang, "Face Recognition Technology based on Kalman Filter," Web of Proceedings, vol. 03, 9th International Conference on Management and Computer Science (ICMCS 2019), 2019, doi: 10.25236/icmcs.2019.003.

[44] H. S. Jang, M. S. Muhammad, and T. S. Choi, "Removal of jitter noise in 3D shape recovery from image focus by using Kalman filter," Microscopy research and technique, vol. 81, no. 5, pp. 207-213, 2017, doi: $10.1002 /$ jemt.22966.

[45] T.-K. Kim, I. Song, and S.-H. Lee, "Noise Reduction of HDR Detail Layer Using a Kalman Filter Adapted to Local Image Activity," Journal of Korea Multimedia Society, vol. 22, no. 1, pp. 10-17, 2019, doi: $10.9717 / \mathrm{kmms} .2019 .22 .1 .010$.

[46] A. Rahiche and M. Cheriet, "KFBin: Kalman filter-based approach for document image binarization," in International Conference on Image Analysis and Recognition, Springer, 2019, pp. 150-161, doi: 10.1007/978-3030-27202-9_13.

[47] E. Hamuda, B. Mc Ginley, M. Glavin, and E. Jones, "Improved image processing-based crop detection using Kalman filtering and the Hungarian algorithm," Computers and Electronics in Agriculture, vol. 148, pp. 37-44, 2018, doi: 10.1016/j.compag.2018.02.027.

[48] N. Q. Ann, D. Pebrianti, Z. Ibrahim, L. Bayuaji, and M. F. M. Jusoh, "Image template matching based on simulated Kalman filter (SKF) algorithm," Journal of Telecommunication, Electronic and Computer Engineering (JTEC), vol. 10, no. 2-7, pp. 37-41, 2018.

[49] M. Eknes and G. Evensen, "An Ensemble Kalman filter with a 1-D marine ecosystem model," Journal of Marine Systems, vol. 36, no. 1, pp. 75-100, 2002, doi: 10.1016/S0924-7963(02)00134-3.

[50] A. Becker, "Kalman Filter Overview," [Online]. Available: https://www.kalmanfilter.net/default.aspx [accessed 12th December, 2019].

[51] M. Fuentes, M. Vivar, J. M. Burgos, J. Aguilera, and J. A. Vacas, "Design of an accurate, low-cost autonomous data logger for PV system monitoring using Arduino ${ }^{\mathrm{TM}}$ that complies with IEC standards," Solar Energy Materials and Solar Cells, vol. 130, pp. 529-543, 2014, doi: 10.1016/j.solmat.2014.08.008.

[52] I. Maxim Integrated Products, "MAX6675: Cold-Junction-Compensated K-Thermocouple-to-Digital Converter $\left(0{ }^{\circ} \mathrm{C}\right.$ to $\left.+1024{ }^{\circ} \mathrm{C}\right), "$ Maxim Integrated Products Inc. [Online]. Available: https://datasheets.maximintegrated.com/ en/ds/MAX6675.pdf. 